\title{
Effect of a yoga intervention on hypertensive diabetic patients Nisha Shantakumari, ${ }^{a *}$ Shiefa Sequeira, ${ }^{\mathrm{b}}$ Rasha Eldeeb ${ }^{a}$ \\ ${ }^{a}$ Department of Physiology, Gulf Medical University, Ajman, United Arab Emirates \\ ${ }^{b}$ Fetal Medicine genetic center, Dubai health care city, United Arab Emirates
}

\section{Accepted on \\ March 19th, 2012 \\ DOI Name \\ 10.3126/jaim.v1i2.6526 \\ Keywords \\ blood sugar, hypertension, type 2 diabetes, yoga \\ Citation \\ Shantakumari $N$, Sequeira S, Eldeeb R. Effect of a $f$ Yoga Intervention on Hypertensive Diabetic $P a-$ tients. Journal of Advances in Internal Medicine 2012;01(02):60-3.}

\begin{abstract}
Background - The concept of psychosomatic medicine has gained popularity, with yoga racing ahead in the field. The present study was conducted to assess the effectiveness of yoga as an intervention in the management of patients with Type 2 diabetes mellitus complicated with hypertension.

Method - This study was carried out in 2005 in Medical College Trivandrum, Kerala, India among 100 hypertensive Type 2 diabetics. They were randomized into control and yoga groups. The yoga group practiced yoga daily for one hour and given oral hypoglycemic drugs for 3 months. The control group did not perform yogic exercises but given oral hypoglycemic drugs. Comparisons were drawn between systolic blood pressure (SBP), diastolic blood pressure (DBP), Fasting blood sugar (FBS) and post prandial blood sugar (PPBS) in both the groups at the start and at the end of 3 months. Paired and unpaired tests were performed.
\end{abstract}

Results - After intervention with yoga for 3 months the study group showed a significant decrease in SBP from $141.71 \pm 9.79$ to $132.23 \pm 7.89 \mathrm{~mm} \mathrm{Hg}$, DBP from $90.57 \pm 4.07$ to $85.49 \pm 5.03 \mathrm{~mm} \mathrm{Hg}$ and FBS from $155.86 \pm 60.53$ to $126.63 \pm 40.59 \mathrm{mg} \%$. The reduction in PPBS from $240.31 \pm 79.42$ to $208.74 \pm 73.05$ mg\% was however not proved to be significant statistically.

Conclusion - These findings suggest that diabetics may benefit from yoga's ability to improve the disease status.

\section{INTRODUCTION}

Type 2 Diabetes mellitus (type 2 DM) is not a single disease process but instead, represents a heterogeneous constellation of disease syndromes all leading to a final common pathway-hyperglycemia. Major metabolic abnormalities coexist in type 2 DM each contributing to the hyperglycemic status including deranged lipid profile, insulin resistance of target tissues and finally abnormal islet cell function. ${ }^{1}$ Sedentary lifestyle, physical inactivity and increasing age contribute to the increase in incidence of type 2 DM. $^{2}$

Yoga is an ancient traditional, Indian psychological, physical and spiritual exercise practice that has been studied for several decades for its role in the management of numerous chronic diseases It is a form of physical activity consisting of various postures (Asana), meditation and breathing techniques (Pranayama). ${ }^{3}$ Yoga is generally safe, simple to learn, and can be practiced by even ill, elderly or disabled individuals. ${ }^{4}$ Yoga has been shown to have therapeutic benefits for individuals with a wide range of health conditions. ${ }^{5,6}$ In addition to its role in improving glycemic control, yoga practice for diabetics may reduce their increased cardiovascular risk via other effects as well. ${ }^{7}$

The present study was undertaken to assess the effect of a controlled yoga training (3 months) programme in the management of type $2 \mathrm{DM}$ with hypertension by measuring fasting blood sugar (FBS), post prandial blood sugar (PPBS) and blood pressure before and after yogic treatment.

\section{METHODS}

The randomized parallel trial was carried out from January to May2005 at the Holistic Medicine out-patient department and Diabetic clinic, Medical College Trivandrum, Kerala, India. The study plan was approved by the Ethics Committee of the medical faculty and conducted in accordance with the guiding principles of the Helsinki Declaration. The sample size was calculated assuming the standard deviation of blood pressure as 10 and the effect size as 6 .The power of the test was considered as $80 \%$ and significance level as $5 \%$. Hence the required sample size calculated was 44 and was approximated to 50 in each arm. Hundred patients with Type 2 diabetes and hypertension in the age group of 35-55 years were recruited according to the criteria of National Diabetes Data Group and JNC 7 guidelines. ${ }^{8,9}$

A patient was classified as a hypertensive diabetic if his FBS value was $\geq 126 \mathrm{mg} / \mathrm{dl}$ (7mmol$/ \mathrm{L})$, PPBS $\geq 200 \mathrm{mg} / \mathrm{dl}$ ( $11.1 \mathrm{mmol} / \mathrm{L})$, systolic blood pressure (SBP) $\geq 130 \mathrm{~mm} / \mathrm{Hg}$ and diastolic blood pressure(DBP) $\geq 80 \mathrm{~mm} /$ Hg. Known diabetic patients who were on treatment with sulphonylureas were included in the study. Patients who are pregnant, on long term steroids and those with known retinopathy, nephropathy, and coronary artery disease and cerebrovascular diseases were excluded from the study.

Hypertensive type 2 diabetics in the age group of 35 - 55 years reporting to the Diabetic clinic meeting the inclusion criteria were randomized into two groups based on their number order of visit to the clinic (Figure1). Even numbers formed the control group while odd numbers comprised the experimental group. Recruitment was stopped when the sample size of 50 was met in each group. The control group ( 26 males and 24 females) did not perform yogic exercises during this period but were on treatment with sulfonylureas. The experimental group (26 males and 24 females) reported to Holistic Medicine department. It was administered yogic practices along with sulfonylureas for a period of 3 months.

Detailed history of the patients including variables like age, gender, history of disease-duration and complications, family history of diabetes, dietary history and treatment history-drug, dosage and duration of treatment

\footnotetext{
* Corresponding author

Nisha Shantakumari,

Department of Physiology, Gulf Medical University, Ajman, United Arab Emirates Email address - nisha@gmu.ac.ae
} 


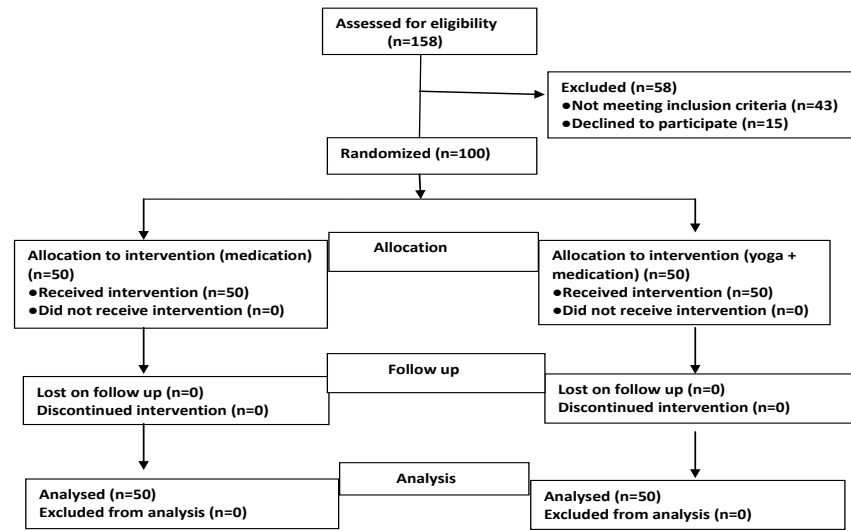

Figure 1. Flow diagram of randomization/ selection of patients

were noted in the questionnaire. Blood pressure was recorded using a mercury sphygmomanometer. Three repeated readings were taken each time and the average was taken as the final reading. FBS and PPBS were estimated following standard techniques by glucose oxidase method. The experimental group was instructed in the Holistic medicine clinic by an experienced yoga teacher in groups of 25 patients each for one hour daily. All patients were taught the same set of yogic exercises, with minor adaptations to the physical abilities of the participants. Yoga treatment as described in Table 1 consisted of practice of (1) asanas (body postures), (2) pranayama (breathing exercises) and (3) meditation

At the end of 2 weeks of supervised yogic training each subject was given advice on ongoing medical treatment and a personalized yoga program to practice regularly at home. They were asked to record the number of minutes they engaged in yoga per day. All subjects of experimental group were required to contact the Holistic Medicine department and the Diabetic clinic once every month for follow-up advice. The control group was to report to Diabetic clinic every month for their follow up after being given advice on ongoing medical treatment at the start of the study. There were no alterations made in the treatment and no changes were recommended in diet of either group during the study period. Both the groups were advised to continue with their carbohydrate restricted fiber rich diet.

The variables of interest were measured at the beginning (day 1) and end (day 90) of the intervention. For the entry of statistical data, the computer package used was Microsoft Excel. All quantitative analyses were conducted in SPSS Version 12 using paired and unpaired sample $t$ tests to compare measures. $P$ value of $<0.01$ was considered highly significant. $P$ value $<0.05$ as significant and $P$ value $>0.05$ was not considered to be statistically significant.

\section{RESULTS}

A total of 52 males and 48 females of mean age 45 years were included in the study (Table 2). All the patients were known to have diabetes for an average duration of 7-10 yrs. There was no significant difference in systolic and diastolic blood pressures between control group and experimental group before intervention.

Table 3 shows the comparison of parameters between control group and experimental group before and after intervention. After practicing yoga for a period of 3 months there was a significant reduction in SBP and DBP of the experimental group. Systolic blood pressure before yoga was in the
Table 1. Yoga practices specified for the experimental group

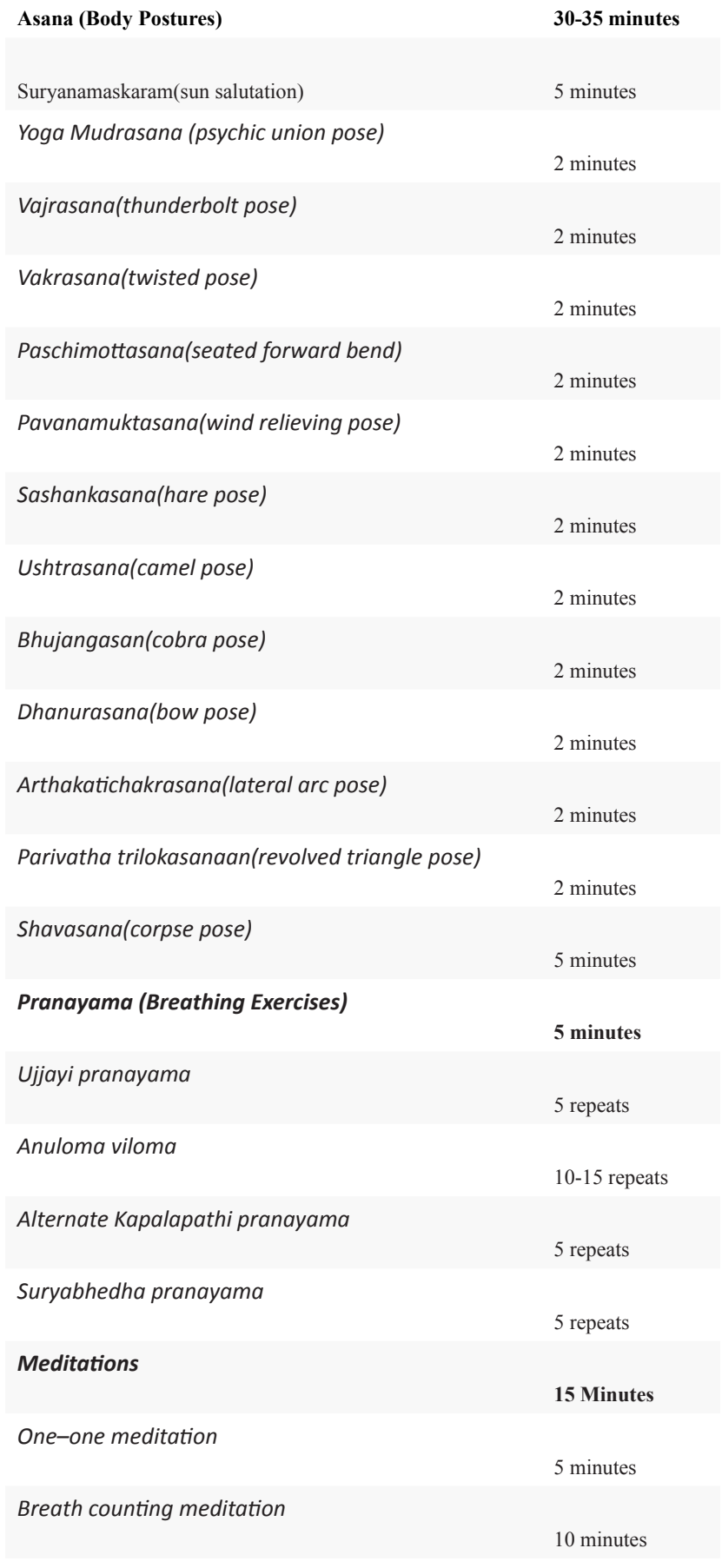

range of 130-160 mm Hg and was reduced to a range of 110-148 $\mathrm{mm} \mathrm{Hg}$. There was a mean reduction in the value by $9.49 \pm 1.90 \mathrm{~mm} \mathrm{Hg}$. DBP also showed a significant reduction in the value by $5.085 \pm 0.978 \mathrm{mmHg}$. The group also showed a significant decrease in FBS at end of 3 months when compared to the values before yogic intervention. Fasting blood sugar before yoga was in the range of $102-240 \mathrm{mg} / \mathrm{dl}$ and was reduced to a range of $89-204 \mathrm{mg} / \mathrm{dl}$. The PPBS showed a mean reduction of $5.54 \pm 6.07 \mathrm{mg} / \mathrm{dl}$. This was however not significant statistically.

Fasting blood sugar and PPBS values of control group remained unchanged from their initial values even after 3 months of medication. However after a period of 3 months the SBP in the control group showed a mean eleva- 
Table 2. Comparison of age and gender distribution and family history between control group and experimental group before intervention

\begin{tabular}{|c|c|c|c|}
\hline Parameter & Group & $\begin{array}{l}\text { Control } \\
\text { Group } \\
\mathbf{N}=\mathbf{5 0}\end{array}$ & $\begin{array}{l}\text { Experimental } \\
\text { Group } \mathbf{N}=\mathbf{5 0}\end{array}$ \\
\hline Age & & $44.46+7.98$ & $45.51+8.89$ \\
\hline \multirow[t]{2}{*}{ Gender } & Male & $26(52 \%)$ & $26(52 \%)$ \\
\hline & Female & $24(48 \%)$ & $24(48 \%)$ \\
\hline Family history & No & $26(52 \%)$ & $14(28 \%)$ \\
\hline
\end{tabular}

tion of $5.03 \pm 16.2$ while the DBP elevated by $2.28 \pm 7.7$.This increase was however statistically insignificant. (Table 3 ) At the end of 3 months there was an elevation in SBP and DBP of the control group, while their FBS and PPBS values showed a reduction. All the parameters of the experimental group showed a reduction from their initial values (Table 3).The reduction in SBP,DBP,FBS and PPBS of the experimental group was however statistically significant when compared to the changes in the control group.

\section{DISCUSSION}

In the present study yoga practice for three months by the study group resulted in a reduction in the FBS, PPBS, systolic BP and diastolic BP. There was an elevation of both systolic and diastolic blood pressures in controls. This was not of statistical significance. In treating hypertension, attention is directed not only to antihypertensives but also to lifestyle modifications. Restriction of calories and alcohol; weight reduction and regular exercise program enhance the control of blood pressure. The absence of regular and strictly followed lifestyle modification may be attributed to be the cause of inadequate control of BP with drug alone in the control group. ${ }^{10}$ Metformin is being used as a cornerstone therapy for Type $2 \mathrm{DM}$ patients. Type $2 \mathrm{DM}$ is characterized by steady loss of $\beta$ cell function and hence deterioration of control of blood glucose. ${ }^{11}$

The decreasing effectiveness of sulphonylureas results not only from progressive loss of $\beta$-cell function but also from patient related factors (dietary incompliance, weight gain, lack of exercise). ${ }^{12}$ As diabetes progresses it becomes increasingly more difficult to attain near-normal glycemic control target levels and more than one agent (i.e., multiple therapies) is required to attain target levels. ${ }^{13}$ These findings could well explain the statistically insignificant improvement in the FBS and PPBS values of the control group. Type 2 DM usually begins in middle life or later. Age and gender standardized prevalence of diabetes showed that females had higher prevalence in india. ${ }^{14}$ In contrast with the Indian data it was found that Pima India males showed greater standardized mortality rates and higher macro vascular complications compared to females. ${ }^{15}$ The two groups in our study were well matched with regard to age, gender and racial status to avoid any confounding factors.

Type $2 \mathrm{DM}$, in contrast to type 1 diabetes is associated with an increased plasma insulin concentration (hyperinsulinemia). However, even the increased levels of insulin are not sufficient to maintain normal glucose regulation because of the greatly diminished insulin sensitivity of the peripheral tissues. The effect of yoga on blood glucose can be postulated
Table 3. Comparison of parameters between control group (Drug only) and experimental group (Drug and yoga) before and after intervention

\begin{tabular}{|c|c|c|c|c|}
\hline Parameters & Control Group & $=50$ & Experimental & Group $\mathbf{N}=\mathbf{5 0}$ \\
\hline & Before & After & Before & After \\
\hline $\begin{array}{l}\text { Systolic blood pres- } \\
\text { sure ( } \mathrm{mm} \mathrm{Hg} \text { ) }\end{array}$ & $137.37(28.77)$ & $142.4(12.57)$ & $141.71(9.80)$ & $132.23(7.89) * *$ \\
\hline $\begin{array}{l}\text { Diastolic blood } \\
\text { pressure (mm Hg) }\end{array}$ & $88.23(17.05)$ & $90.51(9.32)$ & 90.57 (4.07) & $85.49(5.03) * *$ \\
\hline $\begin{array}{l}\text { Fasting blood sugar } \\
(\mathrm{mg} / \mathrm{dl})\end{array}$ & $181.57(66.25)$ & $\begin{array}{l}176.37 \\
(60.47)\end{array}$ & $\begin{array}{l}155.86 \\
(60.53)\end{array}$ & $126.63(40.59) *$ \\
\hline $\begin{array}{l}\text { Post prandial blood } \\
\text { sugar }(\mathrm{mg} / \mathrm{dl})\end{array}$ & $265.31(74.70)$ & $\begin{array}{l}259.77 \\
(60.64)\end{array}$ & $240.31(9.42)$ & $208.74(73.05)$ \\
\hline
\end{tabular}

to result from the beneficial effect on the insulin kinetics. There is improved sensitivity of the target tissues with reduction insulin resistance and consequently, increasing peripheral utilization of glucose. A significant increase in insulin sensitivity, decrease in insulin resistance and a significant rise in the number of insulin receptors by following yogic-intervention was noted by Sahay et al. They also suggested normalization of insulin/ glucagon (I/G) ratio with a reduction in free fatty acid levels. ${ }^{16}$ Stress plays a major role in diabetes mellitus by triggering cortisol which dumps glucose into blood for flight or fright. ${ }^{17}$

Bijlani et al noted that a favorable metabolic response can be produced even by short lifestyle management and stress management programmes. ${ }^{18}$ These could explain significant decline in the FBS values of the experimental group after yogic practice. The reduction in PPBS was however not significant statistically. This could probably be explained on the basis that longer duration of yogic practice might be needed to improve the capacity of body to handle a glucose load of which PPBS is a measure.

The experimental group in this study showed a significant reduction in blood pressure levels both systolic and diastolic after one month of yoga practice and the effect was sustained when studied after 3 months of daily yogic practice. This is in agreement with previous clinical trials which reported changes in blood pressure with lifestyle and /or dietary modifications. ${ }^{19,20}$ Asanas like Paschimottasana when repeated on long term basis have been proved to increase baroreceptors sensitivity and hence effective control of blood pressure. ${ }^{21}$

Psychological stress is a risk factor for hypertension. ${ }^{22}$ Transcendental meditation leading to reduction of stress and decrease in BP in was documented in African American hypertensives..$^{23}$ Various forms of yoga also claim to reduce stress by integrating the body and mind. ${ }^{21,24}$ Other studies evaluating yoga practice suggests that yoga may reduce oxidative stress and endothelial-dependent vasodilatation in patients with established coronary artery disease. ${ }^{25,26}$ Thus we infer that yoga exerts its effect on various facets of blood pressure control. It can combat the causes as well as effects of high blood pressure and stabilize the blood pressure thus reducing hypertension. In conclusion, with the present study yoga practice showed a significant improvement for those diabetic patients with pre-existing complications. Yoga had beneficial effects on BP and blood glucose level. These 
findings suggest that diabetics may benefit from yoga's ability to improve their quality of life.

The yoga package was designed after extensive literature review by yoga specialists and was a perfect combination of asana and breathing exercises targeted at the disease under study. Excellent compliance of study sample. There were no drop outs. Experimental group patients voluntarily reported to Holistic medicine department and were self motivated for the practice of yoga. The control group was also under constant surveillance by the Diabetic care clinic.

The patients could not be kept under direct supervision for the entire period of the study. Dietary data were not recorded regularly. The beneficial effect noted in the experimental group could have been due to the attention given to them and the positive effect of group dynamics during initial period of intervention and could be unrelated to yoga per se. This may lessen the strength of our conclusion. The absence of an active control to allow a control of group experience is also a limitation here.

It can be concluded from this study that the chosen yogic practices are very effective in correcting the hypertension seen in a diabetic patient There is a lot of scope for future work in this field. Having proved the effect of yoga as an adjuvant in treatment of hypertension in diabetics, light is to be thrown on the totally curative value of yoga and also its role in prevention of the disease in potential diabetes.

14. Ramachandran A, Snehalatha C, Kapur. High prevalence of diabetes and IGT in India, National Urban Diabetes survey. Diabetologia 2001;46:1094-101.

15. Wirth RB, Marfin AA, Graw DW et al .Prevalence and Risk factors for Diabetes and Diabetes related amputations in American Indians in South Arizona. Diabetes care 1993;16:354-56.

16. Sahay BK. Role of yoga in diabetes. J Assoc Physicians India 2007; 55: 121-6.

17. Kamei T, Toriniumi V, Kimura H. Decrease in serum cortisol during yoga exercise , correlation with alpha wave activation. Altern the Health Med 2002;36:44-8.

18. Bijlani RL, Vempati RP, Yadav RK, et al. A brief but comprehensive lifestyle education program based on yoga reduces risk factors for cardiovascular disease and diabetes mellitus. J Altern Complement Med 2005;11:267-74.

19. Sacks FM, Svetkey LP, Vollmer WM, et al. Effects on blood pressure of reduced dietary sodium and the dietary approaches to stop hypertension (DASH) diet. N Engl J Med 2001;344:3-10.

20. Appel LJ, Champagne CM, Harsha LS, et al. "Effects of comprehensive lifestyle modification on blood pressure control: main results of the premier clinical trial, JAMA 2003;20:2083-93.

21. Murukeshan R, Govindarajulu N, Bera TK. Effect of selected yoga practices on the management of hypertension. Indian J Physiology and pharmacology 2000;46:487-91.

22. Levenstein S, Smith MW, Kaplan GA. Psychosocial predictors of hypertension in men and women. Archives of Internal Medicine 2001;161:1341-6.

23. Schneider RH, Alexander CN, Staggers F, et al. A randomized controlled trial of stress reduction in African Americans treated for hypertension for over one year. American Journal of Hypertension 2005;18:88-98.

24. Damodaran A, Malathi A, Patil N, et al. Therapeutic potential of yoga practices in modifying cardiovascular risk profile in middle aged men and women. Journal of Association of Physicians of India 2002;50:633-40.

25. Yadav RK, Ray RB, Vempati R, et al. Effect of a comprehensive yoga-based lifestyle Modification program on lipid peroxidation. Indian Journal of Physiology and Pharmacology 2005;49:358-62.

26. Sivasankaran S, Quintner SP, Sachdeva R, et al. The effect of a six-week program of yoga and meditation on brachial artery reactivity: do psychosocial interventions affect vascular tone? Clinical Cardiology 2006;29:393-8. 